\title{
Umbrales en el pensamiento
}

\author{
Roberto Ochoa
}

Universidad Pedagógica Nacional, Cuernavaca, México. Email: rxochoa@yahoo.com

Resumen: Desde una perspectiva que parte de las ideas de Iván Illich sobre la noción de proporcionalidad, el presente artículo hace referencia a la idea de límites o umbrales como un elemento necesario para la reconstrucción de un mundo multicultural. Pero, igualmente bajo la inspiración de Illich, se desmarca de las prácticas científico-profesionales que producen enunciados ajenos a las prácticas populares. Desde afuera y desde arriba no se puede reconstruir "un mundo en el que quepan muchos mundos”. Esto no implica que la búsqueda de nuevos paradigmas sociales sea algo simple. Por el contrario, la profundidad de la búsqueda nos lleva incluso a retomar la vivencia de lo sagrado, como lo describe Mircea Eliade en las sociedades tradicionales, como principio epistémico para ese “otro” mundo posible. mocrático

Palabras clave: Límites, lugar, centro, proporción, Confederalismo De-

\section{Thresholds in thinking}

Abstract: From a perspective that takes place in Ivan Illich's ideas about the notion of proportionality, this article refers to the idea of limits or thresholds as a necessary element for the reconstruction of a multicultural world. But, also inspired by Illich, it takes distance from cientific-professional practices of production of statements that have nothing to do with popular practices. From outside and from the top is not possible to reconstruct "a world where many worlds can coexist". This doesn't mean that the search for new social paradigm is something simple. Nonetheless, the search is so deep that leads us even to reintroduce the experience of sacredness, as described by Mircea Eliade in traditional societies, as an epistemic principle for that "other" possible world.

Keywords: Limits, place, center, proportion, Democratic Confederalism

\section{Limiares no pensamento}

Resumo: Do ponto de vista das idéias em Ivan Illich sobre a noção de proporcionalidade, este artigo refere-se à idéia de limites ou limiares como um elemento necessário para a reconstrução de um mundo multicultural. Mas também sob a inspiração de Illich, diferença-se das práticas científicas e profissionais que produzem declarações alheias das práticas populares. Do lado de fora e de cima não pode reconstruir-se "um mundo no qual caibam muitos mundos". Isso não significa que a busca de novos paradigmas sociais é algo simples. Por outro lado, a profundidade da busca leva a mesmo retomar a experiência do sagrado, como descrito por Mircea Eliade nas sociedades tradicionais, como princípio epistêmico ao "outro" mundo possível.

Democrático

Palavras-chave: limites, localização, centro, proporção, Confederalismo

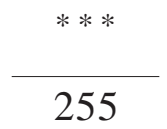


Cerca del final de su vida, Iván Illich dio una serie de entrevistas a David Cayley, las cuales fueron publicadas tiempo después como una especie de testamento intelectual del gran crítico de la sociedad de los servicios, prolongación perversa, esta última, de la sociedad industrial. En algún momento de la conversación, Cayley formuló algo que suena a reproche frente a las posiciones optimistas que Illich había mostrado en la década de los años 70:

"David Cayley: En 1970 sugeriste que las cosas tendrían que cambiar y que, cuando lo hicieran, cambiarían rápidamente.

"Ivan Illich: Estaba equivocado. Cuando menos en el marco de tiempo, estaba equivocado. No pensé que tanta gente pudiera ser tan tolerante al sinsentido. Ahora que estoy de regreso en los EEUU después de 25 años y nuevamente tengo que ver con poblaciones estudiantiles, a veces estoy tan triste que tengo dificultades para dormir (...). No creí que la gente pudiera permanecer tan moralmente tolerante.” (Illich y Cayley 1992: 70)

En su La convivencialidad, el también autor de La sociedad desescolarizada, había escrito, por ejemplo, que escribía el epílogo de la sociedad industrial y que se proponía diseñar los contornos teóricos para una sociedad por venir, una que, esperaba, no fuera hiperindustrial.

Las palabras dramáticas que Illich expresó a Cayley nos hablan de que no podemos confiarnos de las innovaciones cibernéticas que, de hecho, ya habían comenzado a hacerse sentir socialmente en los años 80 y 90 , aunque ahora algunos vean en ellas, particularmente en las redes sociales, el elemento clave para la transformación democrática de la sociedad. Por contraste, si hay un elemento central para la transformación social, es la necesaria intolerancia moral al sinsentido, una intolerancia que nos permita actuar en contra y más allá del avasallamiento de Occidente sobre otras culturas. Pero la necesaria intolerancia moral es algo que se resuelve al interior de las personas y no montados sobre plataformas hipertecnologizadas.

Indígnense, acicateó Stéphan Hessel a la juventud de nuestro tiempo y provocó algo que tal vez no alcanzamos a percibir en toda su magnitud. El grito de este viejo sabio nos está llamando a todos a una reflexión militante, pues -nos dice-sólo cuando uno recupera la faculta humana de la indignación, de la “digna rabia» que dos años antes había sido tema de reflexión de los zapatistas, uno se vuelve capaz del compromiso que la sigue para luchar contra la injusticia. Ahí mora el origen de la transformación social, en un ámbito moral que había estado dormido durante las últimas décadas y que, paradójicamente, quitaba el sueño a Iván Illich.

De esa indignación en movimiento, de las luchas sociales que se despliegan a lo largo y ancho de este mundo malglobalizado, varios colegas nuestros esperan que vayan tomando forma los nuevos paradigmas sociales que nos permitan salir de esta crisis de civilización en todos los senti- 
dos. Abriguemos esperanzas, que, según enseñaba el mismo Illich, es lo único que podemos hacer respecto al futuro.

\section{Los límites: ruptura del paradigma moderno}

En todos lados empieza a reconocerse que la diversidad epistemológica es la última salvaguarda para la preservación del mundo como tal, un mundo en el que siempre han "cabido muchos mundos". Cuando menos, la diversidad se reconoce en el discurso. Incluso, las palabras "multiculturalidad" y "pluriculturalidad" han empezado a tomar vuelo en las últimas décadas ${ }^{1}$. El problema viene, en realidad, cuando de señalar límites se trata, cuando tenemos que reconocer y aceptar que "hasta aquí" llega determinada cultura, lo que nos obliga a respetar a "esta” otra.

Evidentemente, no basta con declararse a favor de la multiculturalidad, si al mismo tiempo se impulsa una pretendida política multicultural con prácticas que son eminentemente occidentales, es decir, propias de una civilización colonizadora.

En agosto del año 2009 publiqué un libro, Muerte al Leviatán, que ha sido muy poco leído y mucho menos incluido dentro de los debates de nuestro tiempo (Ochoa, 2009). No me propongo promoverlo en el presente ensayo, sino sólo retomar algunos argumentos básicos para partir de ellos hacia la formulación de nuevas ideas. En él, reflexiono en torno a la cuestión de la escala de los cuerpos políticos. A la luz de los argumentos de Lepold Kohr, quien fue maestro de Ernst F. Schumacher e inspiró el movimiento "Lo pequeño es hermoso", busco dar continuidad a la urgente revisión de la teoría política moderna, que ha abandonado la consideración de la escala humana como elemento esencial para la actividad política de hombres y mujeres. Los problemas y miserias sociales se desbordan con el tamaño, sostiene Kohr. La respuesta frente a la violencia "no es el incremento del poder policial, sino la reducción del tamaño social”, es decir, del cuerpo político o unidad política fundamental (Kohr 1957). Por mi parte, lo que propongo ahora es que sin la consideración de la cuestión de escala en las ciencias sociales tampoco habrá multiculturalidad posible.

La tesis central que publiqué en 2009 dice que la modernidad occidental se rige por una mentalidad leviatánica, esto es, hechizada por la grandeza y el poder, y que debido a esa mentalidad las ideas de Leopold Kohr, publicadas desde 1957, han sido pasadas de largo e ignoradas por la academia, el mundo intelectual y, por supuesto, los políticos.

La modernidad, para algunos, podrá significar la conquista de la libertad individual frente a los colectivismos de las sociedades tradicionales. Pero este argumento no nos permite explicar la enajenación propia del hombre moderno, tan atado, como nunca antes, al destino de lo que Illich llamó la mega máquina. ¿No será más bien que la pseudo-libertad que conocemos en el mundo moderno no es más que una exacerbación de los 
sentidos que oculta el progresivo y acelerado engrandecimiento de las sociedades, todo, por supuesto, con miras a la acumulación de capitales?

Más allá de un supuesto aumento de la libertad, lo que sí es factible verificar como un hecho de la modernidad es la progresiva acumulación de un poder mayor cada vez en menos manos, las de los dueños de la tecnología. Desde los albores de la modernidad hasta los inicios del siglo XXI el poder no ha dejado de unificarse. Incluso ahora, con las progresivas reformas eficientistas de la ONU, estamos a un paso de lo que en otros tiempos fue sólo ciencia ficción: la conformación del gobierno mundial único.

El camino de las sucesivas concentraciones del poder, propio del orden social moderno, se puede explicar a partir del surgimiento, a lo largo del siglo XVII, de una nueva visión cosmológica y antropológica. La gran transformación de la vida social y política de los siglos XVII y XVII estuvo precedida por una revolución científica, gracias a la cual una nueva visión del mundo tuvo lugar. Por ella, la idea del orden ya no corresponde al orden tal como se percibía en los tiempos premodernos. Con ella, se abre la puerta para la instauración del poder puro y duro, ese que rompe la antigua tradición de la auctoritas: el Leviatán. Su proceso histórico de conformación tiene como contexto principal la concepción de un orden que se instaura por la fuerza y, como matriz, la visión técnica de un poder que se ejerce desde afuera y desde arriba sobre una masa inerte. El proceso de integración y unificación del Leviatán sólo lo pudo dirigir el entendido de que tal poder se ejercería como la aplicación de una fuerza desde el exterior de la sociedad para garantizar la seguridad y tranquilidad, primero, de los súbditos, después, de los clientes-ciudadanos. Esa fuerza utilizaría como principal instrumento un aparato administrativo con el fin de estabilizar los intereses cambiantes y en conflicto de los distintos individuos y grupos sociales. Es por eso que desde los mismos tiempos de Hobbes se habla en términos institucionales de Estado-máquina, Estado-aparato, Estado-mecanismo y Estado-administración.

El punto de quiebre que conduce de la llamada Edad Media a la Modernidad se halla necesariamente en lo que se conoce como revolución copernicana ${ }^{2}$ o revolución científica. El Leviatán de Tomás Hobbes, publicado en 1651, es impensable si no es como parte de esa revolución científica que transformó radicalmente la forma como percibimos el mundo.

Durante la primera mitad del siglo XVII se fue gestando en Europa una escuela de pensamiento comprometida con la sustitución del aristotelianismo, que incluía al tradicional sistema ptolemaico geocéntrico, como marco cosmológico para la filosofía de la naturaleza. Al haber desplazado a la tierra como centro del universo, la revolución copernicana llevaba en sí toda la carga simbólica de un mundo que a partir de entonces no tendría pies ni cabeza. El desarrollo del pensamiento que, paulatinamente, fue elaborando una nueva representación de la realidad a partir de los cálculos de Copérnico minó, al mismo tiempo, la coherencia de la física aristotélica, para la cual la premisa de un cosmos geocéntrico era funda- 
mental. Digamos que los daños causados al armazón aristotélico por el copernicanismo fueron irreparables y esto orilló a los filósofos a construir nuevos discursos sobre la naturaleza. Ese proceso de reconciliación entre la astronomía copernicana y el resto de las ciencias es lo que generalmente conocemos como revolución científica moderna o, también, revolución mecanicista.

La construcción de la nueva teoría sobre la naturaleza no fue una tarea nada sencilla, pues de lo que se trataba, en el fondo, era de explicar el movimiento de la materia prescindiendo del sentido común y de las magníficas explicaciones aristotélicas tradicionales. Para sustituir al sentido común, los autores modernos descubrieron en las primeras máquinas la metáfora perfecta para explicar ahora el funcionamiento del mundo.

Para lograr su objetivo, los principales autores del siglo XVII sometieron a severas críticas las ideas animistas sobre la naturaleza y revivieron antiguas filosofías corpusculares, aunque adaptadas a un contexto cristiano. Una nueva cosmología atomista fue lo que permitió la idea de un espacio infinito con numerosas tierras y soles diseminados. Esta concepción borraba, sin embargo, la tradicional dicotomía cielo-tierra que constituía el marco conceptual básico de toda la filosofía y pensamiento clásicos. Si la tierra era un planeta más, girando alrededor del sol, entonces dejaba de ser plausible considerar al cielo y a la tierra como realidades esencialmente diferentes. Así, en la tierra debían seguirse las mismas leyes que se podían deducir del comportamiento de los astros en el cielo. Según los atomistas, la materia, de idéntica naturaleza en todas partes, estaba sometida al mismo conjunto de leyes que rigen en todos y cada uno de los puntos del espacio infinito y neutro.

La idea del movimiento por inercia en un espacio neutro se erigirá como principio tanto de la física celestial -concebida ahora como sistema solar mecánico- como de la terrenal. La filosofía mecánica se refiere, finalmente, a la teoría que sostiene que todos los fenómenos naturales pueden explicarse en términos de materia corpuscular y movimiento inercial. La ley del movimiento inercial proclama que cualquier corpúsculo en estado de reposo, en medio del espacio, permanece así indefinidamente, a menos que lo empuje otro que, en su respectivo movimiento inercial, choque con él y provoque desde afuera el movimiento del primero. Es una ley que se propone sustituir los movimientos naturales de la física tradicional por la idea de movimiento producido exclusivamente por una fuerza externa.

Para Hobbes, que retoma los principios de la física moderna, todo movimiento se produce exclusivamente por la acción de causas materiales y eficientes. La relación de causalidad es, primero que nada, una relación entre dos cuerpos, uno llamado "agente” y el otro “paciente”, donde el primero actúa desde afuera sobre el segundo, impulsándolo a la inercia. En Hobbes la causalidad ha perdido toda teleología. Así, la metáfora de la máquina se adapta perfectamente a la concepción de un mundo puesto en marcha por un artífice soberano, por un agente sobre el paciente, quien 
desde afuera y desde arriba establece las leyes de su funcionamiento. En esta visión del mundo Dios opera el universo desde afuera, de la misma manera en que un relojero echa a andar el reloj mecánico. El poder de Dios es, entonces, la causa eficiente de un mundo compuesto exclusivamente de materia pasiva, dispuesta a ajustarse según los dictados del técnico.

A esta visión, Hobbes le añade la concepción, también mecanicista, de la constitución social. El cuerpo político, al igual que una máquina y al igual que el mundo entero, estaría compuesto por material pasivo, seres atómicos -minúsculos e iguales-, unidos por el temor a la muerte violenta y gobernados desde arriba y desde afuera por la fuerza de un soberano todopoderoso. El Leviatán es un ordenamiento artificial de partes individuales, ligadas entre sí no por cohesión interna, como en una comunidad orgánica, sino por el temor a la fuerza y la amenaza del castigo.

Tras el derrumbe de la dicotomía cielo-tierra, el mundo moderno emerge entonces bajo el régimen de la igualdad, sobre un espacio neutro y homogéneo. Todo es uno y lo mismo, nada encuentra en la diferencia, y en su relación con lo otro, el fundamento de su ser.

La física aristotélica, en cambio, dependía de una clara diferenciación entre el cielo y la tierra. El lugar en que se ubicaban los cuerpos resultaba radicalmente distinto del "espacio" neutro e infinito propio de la física newtoniana. El relato aristotélico de la naturaleza, íntimamente ligado a su definición del movimiento, tenía como una de sus piezas centrales el concepto de lugar (Lang 1998). El lugar, al ser el primer límite del cuerpo contenido en él, se equiparaba al concepto de forma. Siendo que la forma, por su parte, coincidía también con el fin hacia el cual los cuerpos se dirigen por su propia naturaleza, y que el cosmos estaba dotado de direcciones intrínsecas en cuanto al arriba, abajo, izquierda, derecha, frente y espalda, se desprende la idea de que el lugar representaba también un principio de determinación del movimiento; todas las cosas dentro del mundo se encontraban determinadas con respecto al lugar que les correspondía. Si tomamos en cuenta que cada elemento dentro del cosmos poseía una inclinación específica, esto es, que cada uno de ellos se encontraba ordenado hacia su lugar, este último era entonces legítimamente considerado como principio causal del movimiento. Cada elemento se movía, como de igual a igual, hacia su lugar apropiado y por él. Así, en la relación entre lugar y elemento, se apreciaba claramente una teleología propia de la naturaleza. Si en este mundo "la naturaleza es en todos lados causa de orden", según la famosa máxima aristotélica, esto se debía precisamente a que el lugar, como principio constitutivo formal, volvía al cosmos direccional, en tanto que lo "arriba”, lo "abajo", etcétera, tenía sentido y fundamento como realidad auténtica. Recordemos que para Aristóteles el cosmos era finito y que, por ello, sí tenía un auténtico centro que fungía como principio referencial para los distintos lugares.

Para la física newtoniana, surgida de la revolución científica del siglo XVI, cualquier cuerpo está en el espacio y se mueve a través de él, pero 
el lugar relativo no ejerce la más mínima influencia ni sobre el movimiento ni sobre el reposo. El espacio es un medio completamente pasivo. Se trata de un sustrato inerte -de ahí la "inercia”- que permite el ejercicio, sobre sí, de todos los cuerpos, indistintamente de sus cualidades.

De transformaciones conceptuales, físicas y metafísicas, tan hondas como las ocurridas durante el siglo XVII, tenía que surgir también otra comprensión de la moral y, por supuesto, de la política: la mentalidad leviatánica.

Por toda esta reflexión previa (que se puede consultar a mayor profundidad en mi libro ya mencionado) en el coloquio "Hacia la construcción de un nuevo paradigma social”, convocado por la Universidad Autónoma Metropolitana-Unidad Xochimilco en marzo de 2012, decidí traer a colación los estudios antropológicos bastante conocidos de Mircea Eliade, a partir de las siguientes preguntas.

¿En dónde termina una cultura y empieza otra? ¿Cómo se puede hacer para señalar límites, fronteras, lugares de respeto de un grupo cultural a otro? Tanto para Leopold Kohr como para Iván Illich el concepto de "límite" es fundamental. Pero, al mismo tiempo, su proceder intelectual, su mentalidad, no es leviatánica. Es decir, no llaman a colocarnos, como reputados científicos modernos o como hacen los profesionales, por arriba y por encima de la sociedad, para decretar los límites. Los límites no pueden ser impuestos por el poder, sino vividos por el común. Para que esto sea así, la diferenciación de lugares y la re-ubicación de "centros” cósmicos resulta fundamental: es el primer paso epistémico para romper con el paradigma moderno.

Mircea Eliade identificó claramente que el mundo moderno es aquél en el que el espacio es homogéneo y del cual se han borrado los linderos que separan lo sagrado de lo profano. Si "linderos" es una palabra más suave que la palabra "límites" es porque pone el acento en "lo otro" con lo que linda, más que en la restricción a profanarlos. Sin embargo, linde y límite son sinónimos. Se trata de que para que un linde siga existiendo, tiene que ser respetado, se tiene que constituir como un límite a la profanación. De tal modo, para que podamos refundar "un mundo en el que quepan muchos mundos”, tenemos que recuperar la experiencia de lo sagrado.

El mundo premoderno estaba habitado por una humanidad esencialmente religiosa que hacía diferencia entre los lugares sagrados y, por eso, "fuertes", significativos, y los lugares profanos, no consagrados y, "por consiguiente, sin estructura ni consistencia, en una palabra: amorfos" (Eliade, 1994: 25). La hierofanía, la irrupción de lo sagrado para destacar un territorio del medio cósmico circundante, que se manifestaba en una roca, una montaña, un árbol (o un águila devorando a una serpiente sobre un nopal), descubría un punto fijo que se constituía como eje central, el Axis mundi o pilar cósmico, de toda orientación futura. El foco de la experiencia en las sociedades tradicionales se encuentra, entonces, no en los límites o lindes, 
sino en el centro cósmico alrededor del cual se extiende el "Mundo", "nuestro mundo", precisa Eliade. El lugar sagrado es el primero de los conceptos detrás del cual se encadena el "sistema del mundo" de las sociedades tradicionales, pues "constituye una ruptura en la homogeneidad del espacio" (Eliade, 1994: 38).

Si recordamos aquí a las sociedades tradicionales no es porque pretenda que nos petrifiquemos en una especie de pasado bucólico. Sólo digo que si vivimos el presente, pero pensando en lo sagrado, los límites y las formas sociales serán una consecuencia natural. Los nucleamientos colectivos que surjan harán por sí mismos la diferencia entre lo de adentro y lo de afuera. Los límites no serán impuestos por entidad soberana alguna, no serán fronteras infranqueables, sino umbrales que nos permitirán asomarnos a los otros mundos que, aunque afuera, nos mostrarán, como en un espejo, nuestra propia sacralidad.

\section{Tejiendo de nuevo los hilos}

Los paradigmas sociales, si queremos que sean nuevos y alternativos a la modernidad occidental, no pueden ser sistemas diseñados a la perfección. La reflexión sobre los límites al crecimiento de todo cuerpo social, llevada a cabo primero por Leopold Kohr y después por Ernst F. Schumacher e Iván Illich, es la antípoda de la arrogancia científica que pretende detentar la palabra final sobre los problemas sociales. Los límites, en el pensamiento de Kohr, no representan nunca un decreto final. Este filósofo y economista nacido en Salzburgo inaugura, según Illich, la disciplina de la morfología social, la cual establece una relación proporcional entre el tamaño y la forma de los cuerpos políticos. Sin embargo, no por ello se puede decir que Kohr pretenda erigirse en escultor de la sociedad. La forma social no es delimitable a la manera de los objetos que creamos, delimitándolos instrumentalmente desde el exterior. En lo que concierne a los instrumentos y a los objetos de nuestra industria, nosotros somos los maestros y lo manifestamos teniéndolos, controlándolos y dirigiéndolos con nuestras manos. La sociedad, en cambio, en todo momento nos engloba, nos contiene, y esta compenetración es la que nos impide considerar la forma de la sociedad como si estuviéramos situados fuera de ella. Por contraste, el Leviatán es justamente esa ficción literaria y filosófica acerca de un mecanismo de exteriorización que pretende dirigir el hecho social. Es la metáfora de todo lo que pretende crear el hecho social y darle forma, o simplemente administrarlo, desde afuera y desde arriba, así que de esta pretensión es de la que tenemos escapar. Por eso, al romper con el paradigma moderno, no debemos pretender sustituirlo con otro igual de totalitario.

Esto no impide, ciertamente, que nos movamos en la búsqueda de conceptos que puedan servir de modelo a las aspiraciones de una sociedad que anhela, urgentemente, salir de la crisis de civilización en que, de modo particular, la economía capitalista nos ha sumergido. 
Como lo mencioné arriba, las ideas de Leopold Kohr, así como el estudio de morfología social a que él dio inicio, han sido ignorados. Sin embargo, en un capítulo de libro titulado "La sabiduría de Leopold Kohr" (Illich 2004), Iván Illich sostienen que el tiempo de Kohr llegará cuando la fe en el homo economicus deje su lugar a un escepticismo penetrante y los teóricos sociales lean cuidadosamente a este modesto, pero importante pensador.

Las ideas de Kohr inspiraron a Ernst F. Schumaher para escribir un libro que resultó bastante popular: Lo pequeño es hermoso (Schumacher 1979). Sin embargo -continúan Illich en su análisis -, Kohr sigue siendo un profeta aún para quienes han cultivado la intuición de que lo pequeño es hermoso pero no han descubierto, suficientemente, que la verdadera belleza y bondad no es cuestión tanto de tamaño sino de proporción.

Aunque la palabra "proporción” existe en nuestro lenguaje cotidiano, podemos decir que en el contexto en que la usa Illich, derivada de la teoría musical, es nueva. Cuando habla de proporción, hace referencia al concepto griego de logos, que al mismo tiempo puede significar palabra, equilibrio, definición o proporción. En específico, considera que la definición más precisa de la proporción sería: lo apropiado de una relación (Illich 2004: 237).

La tesis central de Illich dice que cien años antes de la Revolución Francesa, durante el siglo XVII, se habría roto una gran tradición de la civilización occidental que basaba sus principios de orden en una noción bien extendida y explicada culturalmente de la proporción. La proporción era claramente percibida por los sentidos. Tanto para los antiguos como para los medievales existe una proporción, por ejemplo, entre el oído y el sonido, entre el ojo y los colores. Esto quiere decir que los órganos respectivos se encuentran en relación de correspondencia, de proporción, con los elementos del mundo. Si el ojo ve, si el oído oye y si la mano toca, esto se debe a que existe una co-naturalidad, una conmensurabilidad, entre el mundo y los sentidos, y esa era parte de la experiencia de todos los días. La proporción fungía entonces como noción guía y principio de orientación en el $\operatorname{cosmos}^{3}$. De esta manera, la pérdida de la noción de proporción en el siglo XVII implica "la pérdida de los sentidos", y también la pérdida de un mundo. Significa el abandono de un modo de experimentar, para el cual ya no tenemos palabras hoy en día.

Los estudios de Illich no llegan a hacer la relación entre la pérdida de lo que llama "la gran tradición de la proporcionalidad” y la instauración de la monstruosidad del Leviatán como principio de regulación y orden en el mismo siglo. Pero, evidentemente, hay una relación directa.

Lo que Illich sí registra es que, como parte de esa gran tradición de la proporcionalidad, la correspondencia o proporción entre arriba y abajo, derecha e izquierda, macro y micro en el cosmos, fue reconocida intelectualmente como principio de orden, idea que posteriormente fue sustituida por la fabricación de principios de regulación universal. La proporción, 
concluye Illich, fue una estrella polar para la experiencia del propio cuerpo y de la relación con el otro, esto hasta que la monstruosidad de un ordenador -el Leviatán, agrega el que escribe estas líneas- se implantó como requisito necesario del buen vivir.

Para la tradición, entonces, el cosmos significó ese orden de relaciones en el que no sólo los sentidos corresponden con el elemento de su percepción, sino, más allá, en el que todas las cosas están originalmente posicionadas unas con respecto a otras y en armonía con el principio de todo. Kosmein significaba alinear ejércitos, alinear las orillas de un río o emparejar el cielo y la tierra. Para todo pensamiento clásico, la múltiple relacionalidad del cosmos, esa tensión o inclinación de las cosas, una con la otra, comienza con la relación del cielo con la tierra. Según Aristóteles, el cosmos en sí mismo emerge cuando los elementos imitan a los cielos. (Lang 1998: 9). Para los pensadores clásicos y medievales es una certeza que lo que está aquí y lo que está más allá guardan relación entre sí. Es como si fueran una pareja de baile en la cual el movimiento de uno provoca el del otro. No son iguales, sólo que entre ellos hay una liga que los une. El cielo es, de cierto modo, un reflejo de la tierra. Kosmein significa, de hecho, que cielo y tierra son un par. En un mundo así las cosas nunca son por sí mismas, sino que lo son porque algo inevitablemente les corresponde. Todo lo que es, es en relación a algo más. El hombre es hombre por la mujer y viceversa, el padre es por el hijo y viceversa, el fruto es fruto de un árbol. Así, nada puede ser pensado, sentido o experimentado a menos que tenga esta correspondencia fundamental.

Aquí es donde podemos comprender cómo la noción de la proporcionalidad y la vivencia de lo sagrado al interior de las sociedades tradicionales actuaron como mancuerna para que los lindes de una cultura frente a otra no fueran establecidos como muros o límites impermeables. Si el cosmos estaba compuesto por una y muchas dualidades complementarias fundantes, entonces lo de adentro y lo de afuera, más que representar una exclusión, mostraban la continuación de una lógica, la de un orden bien pensado que, en todo caso, puede ser comprendido más como uno que nos apela a la apertura y al don, que a la clausura y la privación de los demás.

Podemos imaginar, con fundamento, cómo los límites que contenían y daban forma a un determinado cuerpo social, a un ethnos, no eran fronteras infranqueables, sino pieles de contacto en el ejercicio de la proporcionalidad.

Si tanto los hombres como las mujeres que transitamos sobre la tierra en este tiempo fuéramos capaces, primero, de volver a experimentar lo sagrado y, segundo, de recuperar la noción de proporcionalidad en todas nuestras relaciones, podríamos comenzar con la regeneración social que tanto urge en estos tiempos de crisis. La conformación de una sociedad renovada seguiría entonces la lógica de un progresivo y ascendente nucleamiento colectivo, a contracorriente con la imposición desde afuera y desde arriba de la voluntad leviatánica. 
Ahora bien, los modelos de organización política y social tampoco pueden surgir de una reflexión tan académica como la que hasta ahora he presentado aquí. Rodolfo Stavenhagen mencionó, durante el Tercer Congreso Nacional de Ciencias Sociales en la Universidad Nacional Autónoma de México, celebrado en febrero de 2012, que los nuevos paradigmas tienen que nacer de las luchas de los movimientos sociales. Un poco más de un mes antes, durante el Coloquio de movimientos antisistémicos celebrado en los primeros días de 2012, en la Universidad de la Tierra-Chiapas, Gustavo Esteva había ido más a fondo cuando habló de la investigación militante o insurgente como aquella que puede desafiar radicalmente a la producción institucionalizada de los enunciados con los cuales hemos dejado que otros nos gobiernen. Convencido de lo que Stavenhagen y Esteva sostienen, me dispongo, para terminar la disertación de este ensayo, a reproducir algunos de los conceptos de uno de los militantes más ilustres de los últimos años: Abdullah Öcalan, el líder del Congreso para la Libertad y la Democracia en Kurdistán, preso en Turquía desde 1999, condenado a cadena perpetua por separatismo y terrorismo, y bajo las fundadas sospechas de que está siendo envenado con estroncio.

El también antiguo jefe del Partido de los Trabajado del Kurdistán (PKK) es un marxista-leninista converso. A partir de su confinamiento solitario en la isla de Imrali (es el único preso en la isla), en el Mar de Mármara, vivió un viraje intelectual hacia el pacifismo, lo que le condujo a estudiar y asimilar las ideas de la ecología social y el municipalismo libertario de Murray Bookchin. Abandonó entonces el sueño por la independencia de un Estado kurdo y comenzó a enarbolar una salida pacífica de autonomía democrática para los kurdos al interior de Turquía. El 20 de marzo de 2005 nombró Confederalismo Democrático a la nueva ideología de lucha de los pueblos del Kurdistán. El Confederalismo Democrático es una propuesta de organización social de tipo piramidal; en la base, las comunidades hablan, debaten, toman decisiones. De la base hacia arriba, los delegados electos formarán un holgado cuerpo de coordinación. Esta sería precisamente el tipo de organización que permitiría el nucleamiento ascendente y progresivo de lo colectivo en una sociedad renovada.

Para entender más puntualmente la diferencia entre un federalismo y un confederalismo, sirve recurrir a la distinción jurídica que el constitucionalista mexicano Felipe Tena Ramírez hace al respecto: "En la confederación los estados que la integran conservan su soberanía interior y exterior, de suerte que las decisiones adoptadas por los órganos de la confederación no obligan directamente a los súbditos de los estados, sino que previamente deben ser aceptadas y hechas suyas por el gobierno de cada estado confederado. En la federación los estados-miembros pierden totalmente su soberanía exterior y ciertas facultades interiores a favor del gobierno central” (Tena Ramírez 1996: 113). Además, en la federación los estados-miembros no ejercen "un dominium sobre el territorio" de su circunscripción (eso que la Constitución mexicana llama la propiedad originaria de la nación), "sino (sólo) un imperium sobre las personas que se encuentran dentro de los límites de su demarcación” (Ibid: 190). De tal 
manera, según Janet Biehl, quien fuera colaboradora habitual de Murray Bookchin, el confederalismo "es el principio amplio de organización política y social que puede institucionalizar la interdependencia, sin recurrir a un Estado, y conservar al mismo tiempo el poder de las asambleas municipales” (Biehl y Bookchin, 1998: 109).

En un texto publicado en 2011 en el que amplía su propuesta de programa político, Abdullah Ocalan dice abiertamente que "el confederalismo democrático es un paradigma social sin Estado” (Öcalan 2011: 21), pero a diferencia del sistema político moderno (Estado o Leviatán), se trata de un sistema no arbitrario, pues es un modelo de acumulación de la experiencia histórica de la sociedad. Mientras que el Leviatan fue mitológica y verdaderamente creado ex nihilo, sirviéndose de un cristianismo secularizado (Ochoa 2009), el confederalismo corresponde con el crecimiento "natural” de las relaciones en sociedad.

Si el Estado moderno es centralista, con miras a favorecer los intereses de poderes monopólicos, en el confederalismo, en cambio, no son los monopolios, sino la sociedad, quien está en el centro de la organización política. De hecho, argumenta Ócalan, “desde tiempos antiguos, la gente siempre ha formado grupos sueltos de clanes, tribus u otras comunidades con cualidades federales". "La estructura heterogénea de la sociedad está en contradicción con todas las formas de centralismo. (...) El modelo administrativo centralista no es un modelo que la gente quiere.” (Öcalan 2011: 23)

Está de más decir que espero que este ensayo sea un acto militante en apoyo a la libertad de Öcalán. Lo que tal vez no está de más decir es que espero que el valor de un insurgente inteligente nos sirva de ejemplo para que la necesaria intolerancia moral frente al sinsentido ascienda como espuma, hasta que logre ahogar y dar muerte al paradigma moderno que tiene atenazada nuestra imaginación. 


\section{Notas}

${ }^{1}$ En México, por ejemplo, se reconoció en 2001 que la nación mexicana tiene una composición pluricultural, esto tras la influencia nacional e internacional que ejerció el movimiento zapatista.

${ }^{2}$ Aunque la revolución copernicana recibe ese nombre porque se inicia con Copérnico, es más bien impulsada por Kepler y culminada por Newton.

${ }^{3}$ Hasta el momento en que Illich escribe, esta desaparición difícilmente ha sido reconocida en la historia de la cultura. Es por ello que no encontraremos esta línea de interpretación en alguna otra teoría o corriente de pensamiento sobre lo que ocurre en el siglo XVII, más que a partir de las investigaciones del propio Iván Illich y sus colaboradores. 


\section{Bibliografía}

Biehl, Janet y Bookchin, Murray (1998), Las políticas de la ecología social. Municipalismo libertario, Colectividad los Arnalejos, Virus editorial y Fundación Salvador Seguí, Bilbao.

Eliade, Mircea (1994), Lo sagrado y lo profano, Labor, 9a . edición, Hamburgo.

Illich, Iván y Cayley, David (1992), Ivan Illich in conversation, Anansi, Ontario.

Illich, Iván (2004), “La sagesse de Leopold Kohr”, en La perte des sens, Fayard, Paris.

Kohr, Leopold (1957), The Breakdown of Nations, Routledge and Kegan Paul, Londres y Nueva York.

Lang, Helen S. (1998), The Order of Nature in Aristotle's Physics. Place and the Elements, Cambridge University Press, Nueva York.

Öcalan, Abdullah (2011), Democratic Confederalism, International Initiative Edition, Cologne, http://www.freedom-for-ocalan.com

Ochoa, Roberto (2009), Muerte al Leviatán. Principios para una política desde la gente, Jus, México.

Schumacher, Ernst Friedrich (1979), Lo pequeño es hermoso. La economía como si la gente importara, H. Blume, Barcelona.

Tena Ramírez, Felipe (1996), Derecho constitucional mexicano, Porrúa, $30^{\mathrm{a}}$ edición, México. 